Theory and si mul at i ons of rel at i vi st i c particle notions i $n$ a magnet osoni $c$ shock wave

\begin{tabular}{|l|l|}
\hline $\begin{array}{l}\text { j our nal or } \\
\text { publ i cat i on ti t l e }\end{array}$ & Journal of Pl asna Physi cs \\
\hline vol une & Vol . 72 \\
\hline number & No. 6 \\
\hline page range & pp. $887-890$ \\
\hline year & 2006-12-01 \\
\hline URL & ht t p: //hdl . handl e. net $/ 10655 / 8958$ \\
\hline
\end{tabular}




\section{Journal of Plasma Physics}

http://journals.cambridge.org/PLA

Additional services for Journal of Plasma Physics:

JOURNAL

Email alerts: Click here

Subscriptions: Click here

Commercial reprints: Click here

PHYSICS

Terms of use : $\underline{\text { Click here }}$

\section{Theory and simulations of relativistic particle motions in a magnetosonic shock wave}

SHUNSUKE USAMI and Y. OHSAWA

Journal of Plasma Physics / Volume 72 / Issue 06 / December 2006, pp 887 - 890

DOI: 10.1017/S0022377806005058, Published online: 20 December 2006

Link to this article: http://journals.cambridge.org/abstract_S0022377806005058

How to cite this article:

SHUNSUKE USAMI and Y. OHSAWA (2006). Theory and simulations of relativistic particle motions in a magnetosonic shock wave. Journal of Plasma Physics, 72, pp 887-890 doi:10.1017/ S0022377806005058

Request Permissions : $\underline{\text { Click here }}$ 


\title{
Theory and simulations of relativistic particle motions in a magnetosonic shock wave
}

\author{
SHUNSUKE USAMI ${ }^{1}$ and Y. OHSA W A ${ }^{2}$ \\ ${ }^{1}$ Computer and Information Network Center, National Institute for Fusion Science, \\ 322-6 Oroshi-cho, Toki, Gifu 509-5292, Japan \\ ${ }^{2}$ Department of Physics, Nagoya University, Nagoya 464-8602, Japan
}

(Received 15 August 2005 and accepted 20 December 2005)

\begin{abstract}
The motions of relativistic particles in a magnetosonic shock wave propagating obliquely to an external magnetic field are studied. In the zeroth-order theory, particles continue to move nearly parallel to the external magnetic field in the shock transition region, when the shock speed is close to $c \cos \theta$, where $c$ is the speed of light and $\theta$ is the propagation angle. Perturbations to this zeroth-order motion are also analyzed for positrons and ions. The perturbation frequency of positrons is $\omega \sim \Omega_{\mathrm{p} 0} \gamma^{-1}$ and that of ions is $\omega \sim \Omega_{\mathrm{i} 0} \gamma^{-1 / 2}$, where $\Omega_{\mathrm{p} 0}$ and $\Omega_{\mathrm{i} 0}$ are the non-relativistic gyrofrequencies of positrons and of ions, respectively, and $\gamma$ is the Lorentz factor. These theoretical predictions are confirmed with numerical simulations.
\end{abstract}

\section{Introduction}

Magnetosonic shock waves can accelerate thermal hydrogen ions, heavy ions, and electrons with various non-stochastic mechanisms [1]. Recently, two acceleration mechanisms of non-thermal, relativistic particles in magnetosonic shock waves have been studied with theory and simulations. One mechanism is associated with largeradius gyromotions; particles absorb energy from the electric field perpendicular to the magnetic field $\mathbf{B}[2]$. The other is the acceleration parallel to $\mathbf{B}[3,4]$. Here, we investigate particle motions in the latter. In Sec. 2 , we describe the zeroth-order and perturbation theories for relativistic particles. In Sec. 3, we verify them with simulations. Section 4 gives a summary of our work.

\section{Theory}

\subsection{Zeroth-order theory}

We analyze the motions of relativistic particles in a magnetosonic shock wave propagating in the $x$ direction with a speed $v_{\mathrm{sh}}$ in an external magnetic field $\mathbf{B}_{0}=B_{0}(\cos \theta, 0, \sin \theta)$. If the particle speed $v$ is very close to the speed of light $c$, a slight change in the particle speed can lead to a great change in the Lorentz factor $\gamma$. Therefore, ignoring $\gamma d \mathbf{v} / d t$ compared with $\mathbf{v} d \gamma / d t$, we obtain the zeroth-order equation of motion for a particle with a mass $m_{j}$ and a charge $q_{j}(j$ denotes particle species, $j=$ p or i):

$$
m_{j} \frac{d \gamma_{0}}{d t} \mathbf{v}_{0}=q_{j}\left(\mathbf{E}+\frac{\mathbf{v}_{0}}{c} \times \mathbf{B}\right)
$$

where the subscript 0 refers to the zeroth-order quantities. 
We consider particles moving with the wave, i.e. $v_{x 0}=v_{\mathrm{sh}}$. When $v_{\mathrm{sh}} \approx c \cos \theta$, we find

$$
\frac{v_{z 0}}{v_{\mathrm{sh}}} \approx \frac{B_{z 0}}{B_{x 0}}
$$

from (2.1). It is also shown that $\left|v_{y 0}\right|$ is much smaller than $v_{x 0}$ and $v_{z 0}$; thus, the particles move nearly parallel to $\mathbf{B}_{0}$. Moreover, we obtain the time rate of change of $\gamma_{0}$ as

$$
\frac{d \gamma_{0}}{d t}=\frac{q_{j} B_{x 0}}{m_{j} v_{\mathrm{sh}}} \frac{\left(\mathbf{E} \cdot \mathbf{B}_{0}\right)}{\left(\mathbf{B} \cdot \mathbf{B}_{0}\right)} .
$$

If the particle position in the wave does not change, $\gamma_{0}$ continues to grow linearly with time [3].

\subsection{Perturbation theory}

The zeroth-order theory is applicable to either positrons or ions. We do, however, need to treat positron and ion perturbations separately [4]. We assume that $d \mathbf{v}_{1} / d t \sim \gamma_{0}^{-1} \Omega_{\mathrm{p} 0} \mathbf{v}_{1}$ for positrons and $d \mathbf{v}_{1} / d t \sim \gamma_{0}^{-1 / 2} \Omega_{\mathrm{i} 0} \mathbf{v}_{1}$ for ions, where $\Omega_{\mathrm{p} 0}$ and $\Omega_{\mathrm{i} 0}$ are the non-relativistic positron and ion gyrofrequencies, respectively, and the subscript 1 refers to perturbed quantities. We then expand the exact equation of motion. After some algebra, the perturbation frequency $\omega$ of the positrons is obtained as

$$
\omega^{2}=\left(\frac{e}{m_{\mathrm{p}} c}\right)^{2} \frac{\left(\gamma_{0}^{2} / c^{2}\right)\left(\mathbf{B}_{0} \cdot \mathbf{v}_{0}\right)^{2}+B^{2}}{\gamma_{0}^{4}},
$$

which is obviously positive, while that of the ions is given as

$$
\omega^{2}=-\frac{q_{\mathrm{i}}}{m_{\mathrm{i}} \gamma_{0} \gamma_{\mathrm{sh}}}\left(\frac{d E_{x}}{d \xi_{0}}+\frac{v_{y 0}}{c} \frac{d B_{z}}{d \xi_{0}}-\frac{v_{z 0}}{c} \frac{d B_{y}}{d \xi_{0}}\right),
$$

where $\gamma_{\mathrm{sh}}=\left[1-\left(v_{\mathrm{sh}} / c\right)^{2}\right]^{-1 / 2}, \xi=x-v_{\mathrm{sh}} t$, and $\xi_{0}$ is the center position of the perturbation; $d / d \xi_{0}$ designates the derivative at $\xi=\xi_{0}$. The ion perturbation is stable when $\omega^{2}>0$.

\section{Numerical studies}

We numerically investigate the motions of relativistic particles. For positrons, we use a one-dimensional, relativistic, electromagnetic particle simulation code [3]. As in the theory, waves propagate in the $x$ direction in an external magnetic field $\mathbf{B}_{0}$. The field strength is $\left|\Omega_{\mathrm{e} 0}\right| / \omega_{\mathrm{pe}}=3$, where $\left|\Omega_{\mathrm{e} 0}\right|$ and $\omega_{\text {pe }}$ are the electron gyro and plasma frequencies, respectively. The propagation angle is taken to be $\theta=42^{\circ}$. The propagation speed of a shock wave studied here is observed to be $v_{\mathrm{sh}}=2.4 v_{\mathrm{A}}$, where $v_{\mathrm{A}}$ is the Alfvén speed. It has a typical shock profile with the width of the transition region of the order of the ion inertial length as shown, for instance, in [3]. Figure 1 shows the time variation of $\gamma$ of a positron accelerated by a magnetosonic shock wave in an electron-positron-ion plasma with a positron-to-electron density ratio of 0.02 [3]. The energy increases up to $\gamma \sim 600$. We did, however, find oscillations in $\gamma$. Figure 2 displays the oscillation frequency $\omega$ as a function of $\gamma_{0}$ [4]. The data points represent simulation results, while the solid line shows the theoretical curve given by (2.4). The simulation results are explained by the theory. 


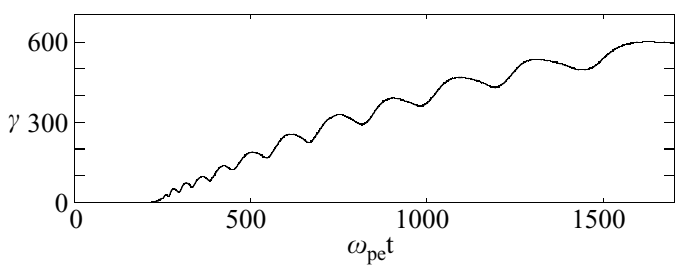

Figure 1. Time variation of $\gamma$ of an accelerated positron. Here, $\omega_{\text {pe }}$ is the electron plasma frequency.

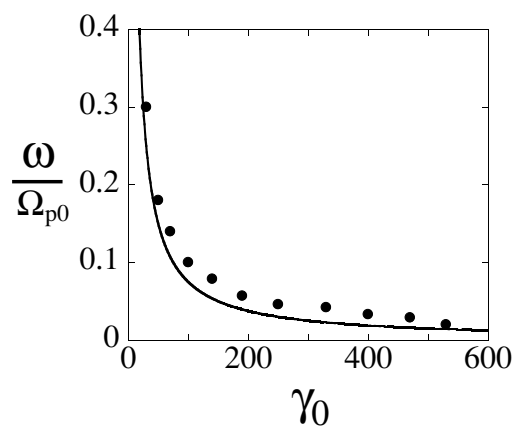

Figure 2. Positron perturbation frequency $\omega$ versus $\gamma_{0}$.

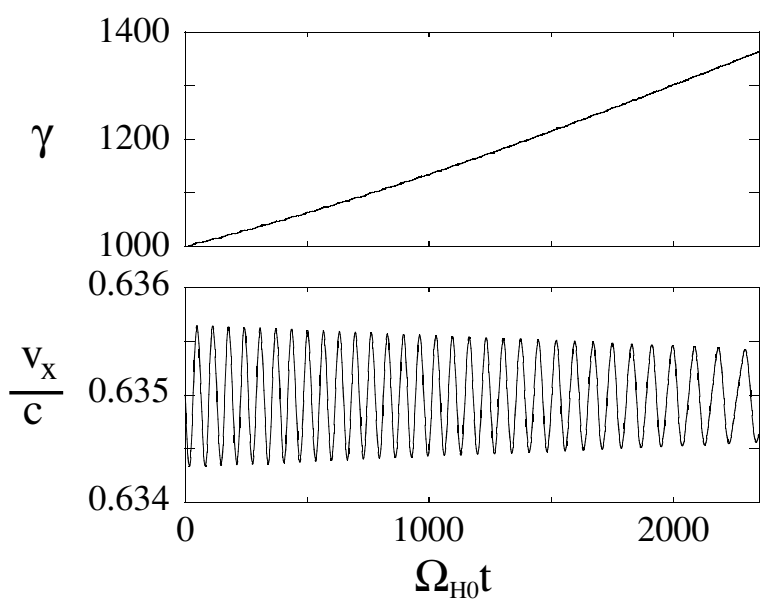

Figure 3. Time variations of $\gamma$ and $v_{x}$ of an accelerated hydrogen ion. Here, $\Omega_{\mathrm{H} 0}$ is the non-relativistic hydrogen gyrofrequency.

For ions, we calculate test particle orbits; we first obtain the electromagnetic fields in a shock wave from a particle simulation and then follow particle motions in the fields, assuming stationary wave propagation. Here, plasma parameters are $\left|\Omega_{\mathrm{e} 0}\right| / \omega_{\mathrm{pe}}=1.5, \theta=50^{\circ}$, and $v_{\mathrm{sh}}=3.2 v_{\mathrm{A}}$. The initial velocities of the test particles are given by the zeroth-order theory. In Fig. 3 we show the time variations of $\gamma$ and $v_{x}$ of an ion with an initial energy $\gamma=1000$. The energy increases from $\gamma=1000$ 


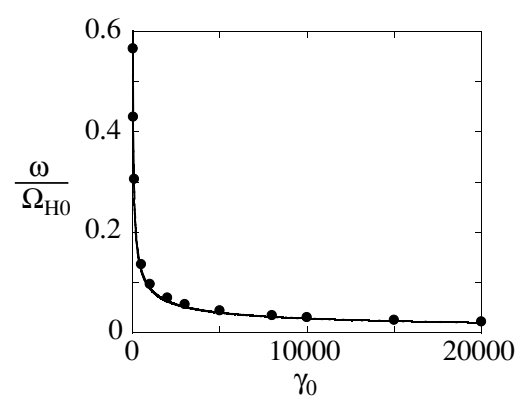

Figure 4. Ion perturbation frequency $\omega$ versus $\gamma_{0}$.

to approximately 1400. Also, we find small-amplitude oscillations in $v_{x}$. In Fig. 4, we display the perturbation frequency $\omega$ as a function of $\gamma_{0}$. The simulation values fit well to the theoretical curve obtained from (2.5)

\section{Summary}

The motions of relativistic particles in a magnetosonic shock wave have been studied. In the zeroth-order theory, where the relation $\gamma|d \mathbf{v} / d t| \ll v d \gamma / d t$ is assumed, particles are accelerated almost parallel to the external magnetic field, when $v_{\mathrm{sh}} \approx$ $c \cos \theta$. This is applicable to either positrons or ions. Perturbation theories for positrons and ions have been separately investigated. The perturbation frequency of positrons is $\omega \sim \Omega_{\mathrm{p} 0} \gamma_{0}^{-1}$ and that of ions is $\omega \sim \Omega_{\mathrm{i} 0} \gamma_{0}^{-1 / 2}$. The zeroth- and first-order theories have been verified with numerical simulations.

\section{References}

[1] Ohsawa, Y. 2004 Nonstochastic particle acceleration in collisionless shock waves. Physica Scripta T107, 32-35.

[2] Usami, S. and Ohsawa, Y. 2004 Evolution of relativistic ions incessantly accelerated by an oblique shock wave. Phys. Plasmas 11, 918-925.

[3] Hasegawa, H., Usami, S. and Ohsawa, Y. 2003 Positron acceleration to ultrarelativistic energies by a shock wave in a magnetized electron-positron-ion plasma. Phys. Plasmas $10,3455-3458$.

[4] Usami, S. and Ohsawa, Y. 2004 Motions of ultrarelativistic particles accelerated in an oblique plasma wave. Phys. Plasmas 11, 3203-3211. 